\title{
NOTULES HYDRAULIQUES
}

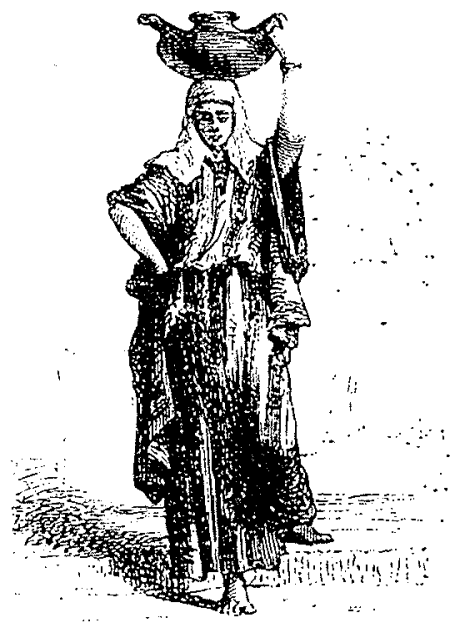

\author{
RADIAL FLOW ON A HORIZONTAL PLANE \\ BY HAROLD R. VALLENTINE, M. A.S.C.E* *
}

\section{Introduction}

Axially symmetrical radial flow of a liquid with a free surface can be analysed as a case of spatially varied flow in a uniform rectangular channel; the channel is considered to extend along a radius, and its discharge decreases inversely as the distance (Fig. 1 a).

Alternatively, radial flow can be analysed as a limiting case of flow in a diverging rectangular channel, the angle between the walls being $2 \pi$. If surface resistance effects are neglected, the surface profiles in any diverging, rectangular channel, with a divergent angle $\theta$ and a steady flow rate $Q_{\theta}$, will be the same as those for axisymmetrical radial flow at a rate:

$$
\mathrm{Q}=\frac{2 \pi}{\theta} \mathrm{Q}_{0}
$$

If surface resistance effects are considered, the profiles differ to the extent that the channel walls contribute to the resistance to flow in the channel, for the axisymmetrical flow has no wall resistance. The wall effect becomes significant, in this respect, if the depth of flow exceeds about five per cent of the channel width.

Most of the literature on channel expansions treats the cases of flow without surface resistance. The general forms of the profiles are well known but it is necessary, in practice, to compute the profile for each set of boundary and flow conditions.

The object of this paper is to present generalised profiles in non-dimensional form both for radial flows without resistance and for radial flows with bed resistance. These profiles can facilitate the solution of problems involving radial flow, either the axisymmetrical or the diverging channel type. It will be seen that the resistance-free assumption does not yield satisfactory approximations to the profiles for supercritical flow of real liquids.

* Professor of Civil Engineering, University of Newcastle, N.S.W., Australia.

\section{Flow without surface resistance}

The possible profiles of axisymmetrical radial flow without surface resistance on a horizontal plane are represented diagrammatically in Figure $1 \mathrm{~b}$. The liquid is so introduced near the axis that the flows is essentially horizontal and at critical depth at the release radius, $r_{0}$.

Under such conditions, free surface flow is not
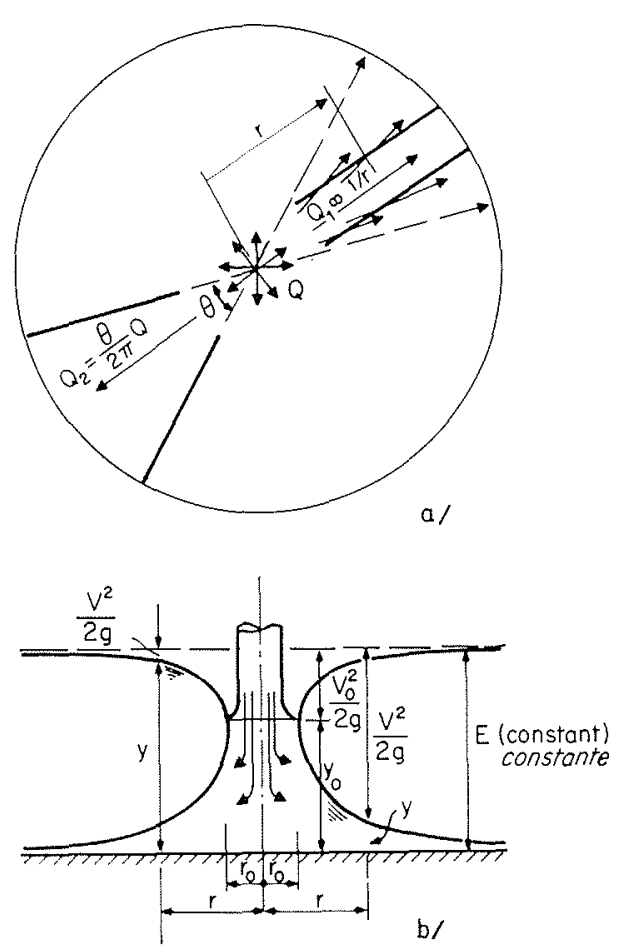

1/ Axisymmetrical radial flow without surface resistance, flow in a diverging channel and spatially varied flow in a uniform channel.

Ecoulement radial et axisymétrique, sans résistance superficielle, écoulement dans un chenal divergent, et écoulement variable dans l'espace, dans un chenal uniforme. 
possible for a radius $r<r_{0}$. For $r>r_{0}$, flow will be either subcritical or supercritical depending upon the downstream control depth. The dimensions on the left of Figure $1 b$, refer to subcritical flow and those on the right to supercritical flow. At a particular radius, the subcritical and the supercritical flows are at the alternate depths.

The specific energy, E, is constant throughout the flow and, if the deptlis and velocities at radii $r_{0}$ and $l$ are $y_{0}, V_{0}$ and $y, V$ respectively,

Also:

$$
\frac{\mathrm{V}^{2}}{2 g}+y=\frac{\mathrm{V}_{0}{ }^{2}}{2 g}+y_{0}=\mathrm{E}
$$

$$
V=\frac{Q}{2 \pi r y}
$$

and, from Equations 1 and 2:

$$
\begin{aligned}
\frac{y}{y_{0}}-1 & =\frac{\mathrm{V}_{0}{ }^{2}}{2 g y_{0}}\left[1-\left(\frac{r_{0}}{r}\right)^{2}\left(\frac{y_{0}}{y}\right)^{2}\right] \\
\therefore \frac{y}{y_{0}}-1 & =\frac{1}{2} \mathscr{H}_{0}^{2}\left[1-\left(\frac{r_{0}}{r}\right)^{2}\left(\frac{y_{0}}{y}\right)^{2}\right]
\end{aligned}
$$

where $\mathscr{H}_{0}$ is the Froude number at $r=r_{0}, \quad \mathscr{H}_{0}$ is unity in Figure 1 since $y_{0}=y_{c}$, the critical-depth at this radius. However, Equation 3 is valid for any value of $r_{0}$ provided it is greater than the radius at which critical depth flow occurs.

When $\mathscr{H}_{0}$ is unity, Equation 3 becomes:

$$
\frac{y}{y_{0}}-1=\frac{1}{2}\left[1-\left(\frac{r_{0}}{r}\right)^{2}\left(\frac{y_{0}}{y}\right)^{2}\right]
$$

which defines the two possible profiles in non-
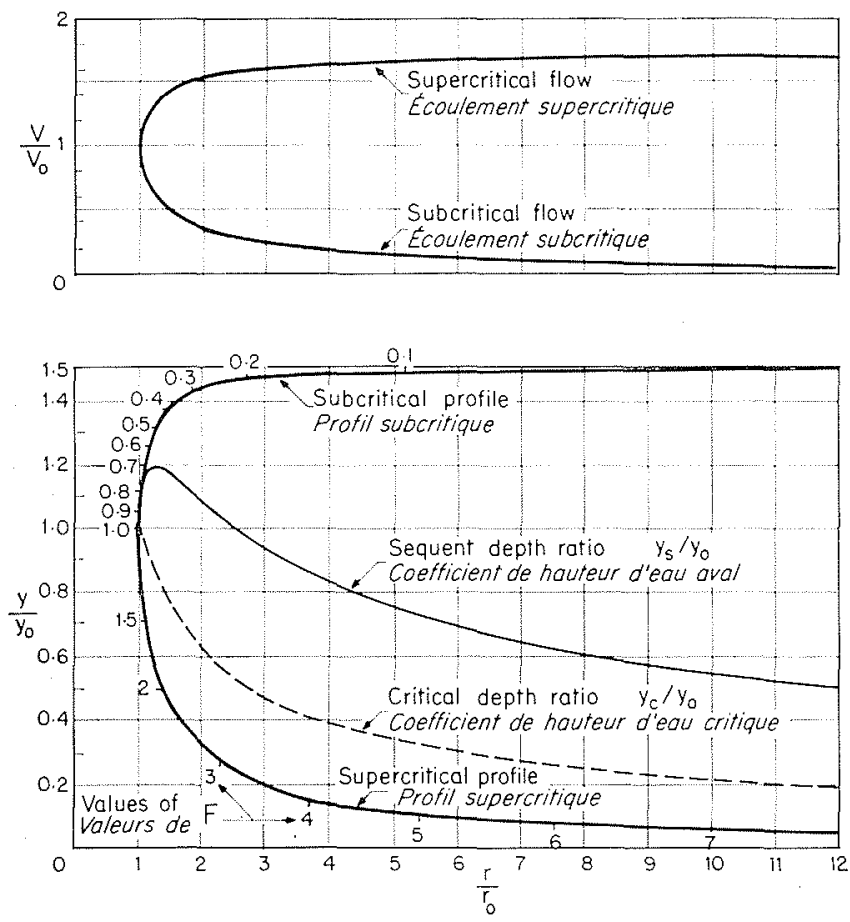

2/ Axisymmetrical, radial flow on a horizontal plane without resistance. Depth and velocity relationships.

Ecoulement radial et axisymétrique sur un plan horizontal, sans résistance. Relations entre les hauteurs d'eau et les vitesses. dimensional terms. These profiles are plotted in Figure 2.

Several features of the profiles are worthy of mention. The subcritical profile is seen to rise to within one per cent of its ultimate value of $1.1 / 2$ times the critical depth at $\left(r / r_{0}\right)=3.9$; on the other hand the depth of the supercritical profile decreases with increase in radius, being one per cent of its initial value at $\left(r / l_{0}\right)=122$.

The variation in velocity follows from Equation 2:

$$
\frac{\mathrm{V}}{\mathrm{V}_{0}}={\frac{r_{0} y_{0}}{r y}}^{2}
$$

The velocity varies only gradually and almost inversely as the radius in the region $\left(r / r_{0}\right)>4$ as shown in Figure 2.

The Froude number at any radius $r$ is:

$$
\mathscr{H}=\sqrt{\frac{V^{2}}{g y}}=\sqrt{\frac{Q^{2}}{4 \pi^{2} g^{2} y^{3}}}
$$

and, since $\mathscr{F}_{0}$, at $\left(r / r_{0}\right)=1$, is unity:

$$
\text { अㄱ }=\frac{r_{0}}{r}\left(\frac{y_{0}}{y}\right)^{3 / 2}
$$

The values of $\mathscr{G}$ are shown adjacent to the profiles in Figure 2.

The critical-depth, $y_{c}$, decreases with increase in radius, for:

whence:

$$
y_{c}=\sqrt[3]{\frac{q^{2}}{g}}=\sqrt[3]{\frac{\mathrm{Q}^{2}}{4 \pi^{2} r^{2} g}} \propto \frac{1}{r^{2 / 3}}
$$

$$
\frac{y_{p}}{y_{0}}={\frac{r_{0}}{r}}^{2 / 3}
$$

The critical-depth curve is shown in broken line in Figure 2. Since it is a function only of $\mathrm{Q}$ and $r$, this curve is applicable to both flows without and flows with surface resistance.

If the supercritical radial flow is terminated by a circular hydraulic jump, with antecedent depth $y_{a}$ and sequent or tailwater control depth $y_{s}$, these depths are related by the ordinary jump equation:

$$
\frac{y_{s}}{y_{a}}=\frac{1}{2} \sqrt{1+8 \mathscr{y}_{a}^{2}}-\frac{1}{2}
$$

if the effect of the length of the jump is neglected. (If the downstream water surface is not horizontal, the effective downstream depth, and hence the jump location, is slightly affected by the length of the jump.) From Equations 9 and 10, the equation of the sequent depth curve, shown in Figure 2 is:

$$
\frac{y_{k}}{y_{a}}=\frac{1}{2} \sqrt{1+8\left(\frac{r_{0}}{r}\right)^{2}\left(\frac{y_{0}}{y}\right)^{3}}-\frac{1}{2}
$$

The location and height of a hydraulic jump, if it occurs, are determined by the intersection of the tailwater profile and the sequent depth curve.

\section{Flow with surface resistance}

The differential equation for the surface profile of axially symmetrical flow on a horizontal plane 
with bed resistance is obtained by differentiation of the total head, $\mathrm{H}$, with respect to radius.

$$
\begin{aligned}
\mathrm{H} & =y+\frac{\mathrm{V}^{2}}{2 g} \\
\therefore \frac{d \mathrm{H}}{d r} & =\frac{d y}{d r}+\frac{d}{d r}\left(\frac{\mathrm{Q}^{2}}{8 \pi^{2} g r^{2} y^{2}}\right) \\
\therefore-\mathrm{S} & =\frac{d r}{d y}-\frac{\mathrm{Q}^{2}}{4 \pi^{2} g r^{2} y^{2}}\left(\frac{1}{r}+\frac{1}{y} \frac{d y}{d r}\right) \\
\therefore \frac{d y}{d r} & =\frac{\left(\mathrm{V}^{2} / g r\right)-\mathrm{S}}{1-\left(\mathrm{V}^{2} / g y\right)}
\end{aligned}
$$

This equation can be derived from the standard equation $\left(^{*}\right)$ for spatially varied flow in horizontal prismatic channel of rectangular cross section with decreasing discharge:

$$
\frac{d y}{d x}=--\frac{\mathrm{Q}_{1} q_{*} / g \mathrm{~A}^{2}-\mathrm{S}}{1-\mathrm{Q}_{1}^{2} / g \mathrm{~A}^{2} y}
$$

where $q_{*}=(d \mathrm{Q} / d x)$, the space rate of change of the flow, $Q_{1}$, in the channel.

If the total radial discharge is $Q$, the flow through an arc of length $B$ at a radius $r$ is:

$$
\begin{aligned}
\mathrm{Q}_{1} & =\frac{\mathrm{QB}}{2 \pi r} \\
\therefore q_{*} & =\frac{d \mathrm{Q}_{1}}{d x}=-\frac{\mathrm{QB}}{2 \pi r^{2}} \\
\therefore \frac{\mathrm{Q}_{1} q_{*}}{g \mathrm{~A}^{2}} & =-\frac{\mathrm{Q}^{2} \mathrm{~B}^{2}}{4 \pi^{2} r^{3} \mathrm{gA}^{2}}=-\frac{\mathrm{Q}^{2}}{4 \pi^{2} r^{3} g y^{2}}=-\frac{\mathrm{V}^{2}}{g r^{*}} \cdots
\end{aligned}
$$

so that Equation (14) reduces to Equation (13).

According to the Chezy formula, $\mathrm{S}=\left(\mathrm{V}^{2} / \mathrm{C}^{2} y\right)$ and Equation (13), expressed as:

$$
\frac{d y}{d r}=\frac{y / r-g / \mathrm{C}^{2}}{1 / \mathscr{G}^{2}-1}
$$

can be converted to a form suitable for numerical computation in terms of $y / y_{0}$ and $r / r_{0}$ as follows:

$$
\frac{d\left(y / y_{0}\right)}{d\left(r / r_{0}\right)}=\frac{\frac{y}{y_{0}} / \frac{r}{r_{0}}-\frac{g}{\mathrm{C}^{2}} \frac{r_{0}}{y_{0}}}{\left(\frac{r}{r_{0}}\right)^{2}\left(\frac{y}{y_{0}}\right)^{3}-1}
$$

It is evident that the profiles depend upon two additional variables $r_{0} / y_{0}$ and $\mathrm{C}$, which were not involved in the resistance-free profiles. The significance of the $r_{0} / y_{0}$ ratio is evident from the expressions for $V_{0}$ :

$$
\mathrm{V}_{0}=\frac{\mathrm{Q}}{2 \pi r_{0} y_{0}}=\sqrt{g y_{0}}
$$

whence:

$$
\mathrm{Q}=2 \pi \frac{r_{0}}{y_{0}} g^{1 / 2} y_{0}^{5 / 2}
$$

Therefore, if $\mathrm{Q}$ and $r_{0} / y_{0}$ are known, $y_{0}$ and also $r_{0}$ can be determined; and for a given $Q$, a large value of $r_{0} / y_{0}$ corresponds to a low value of $y_{0}$.

$\left.{ }^{*}\right)$ CHow (V.T.) : Open Channel Hydraulics, McGraw-Hill (1959), p. 332.

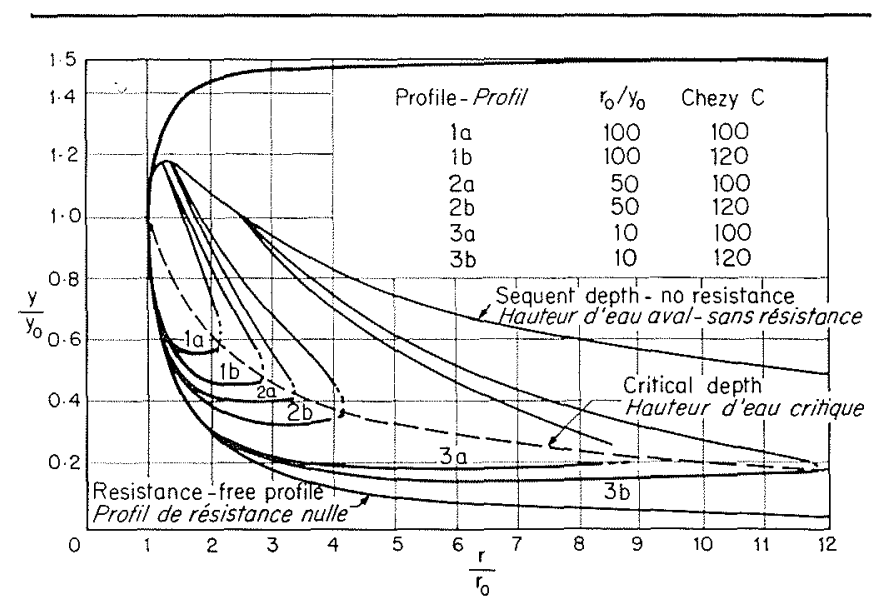

3/ Profiles and sequent depths for radial flows with bed resistance on a horizontal plane.

Profils et hantenrs d'eaul aval, correspondant à des écoulements radianx, avec résistance sur le fond sur un plan horizontal.



4/ Determination of approximate profiles for supercritical radial flow.

Déternination des profils approchés pour l'écoulement radial en régime supercritique.

In Equation 17, the effect of the resistance term $g / \mathrm{C}^{2}$ on the profile is increased by the factor $r_{0} / y_{0}$. Although this effect is small for subcritical flows, it becomes appreciable in supercritical flows, particularly with increasing $r_{0} / y_{0}$ values. When the numerator is zero, the profile is horizontal; and when it becomes negative the profile rises towards the critical depth line, turning vertically to meet it when the denominator becomes zero. These conditions do not arise in flow without resistance.

A selection of supercritical flow profiles based on Equation 17 with constant $\mathrm{C}$ is shown in Figure 3. They are for Chezy's $C$ values of 100 and 120 with initial values of $x_{0} / y_{0}$ of 10,50 and 100 .

The sequent depth curve for each profile is shown in fine line, extending from the sequent depth curve for resistance free flow to the common critical-depth curve. With a knowledge of the tailwater control depth for a particular profile, the location of the circular hydraulic jump can be determined. The co-ordinates of the point on each of the profiles where the depth is a minimum follows from setting Equation 17 at zero. These coordinates define a locus whereby the minimum flow 
depth ratio $y / y_{0}$, can be plotled as a function of $r / r_{0}$ and $r_{0} g / y_{0} \mathrm{C}^{2}$. This plot (Fig. 4) can serve as an approximate guide to the form of a profile given $r_{0} / y_{0}$ and $\mathrm{C}$.

For example suppose that flow is at critical depth in a diverging channel at a section which is 5 feel wide and 20 feet from the effective centre of diver. gence. If the flow rate is $28.4 \mathrm{cfs}$, and the Chezy coefficient for the channel is 80 , the approximate profile can be determined as follows:

The critical depth:

$$
\begin{gathered}
y_{0}=\sqrt[3]{\frac{q_{0}^{2}}{g}}=1 \mathrm{ft} \\
\frac{r_{0} g}{y_{0} C^{2}}=\frac{20 \times 32.2}{1 \times 80^{2}}=0.10
\end{gathered}
$$

The approximate profile is sketched as a horizon- tal line through the point corresponding to $\left(r_{0} g / y_{0} \mathrm{C}^{2}\right)=0.10$, with appropriate end transitions as sketched in Figure 4.

\section{Conclusion}

The characteristics of axisymmetric radial flow over a horizontal plane have been presented in nondimensional form for flows with and withoul bed resistance. The profiles for supercritical flow with bed resistance have been shown to differ appreciably from that for resistance-free flow. An approximate method has been developed for the rapid determination of the profiles for supercritical flows with bed resistance.

\section{ABNORMAL GROUPING OF LARGE EDDIES IN A SUBMERGED JET}

\section{BY S.K.A. NAIB, B.Sc. (Eng.) Ph.D., D.I.C., A.C.G.I. *}

It is well known that when a turbulent jet flows through an enveloping fluid, the surface of discontinuity breaks up into vortices which are carried alternatively along the surface. The development of these vortices in the course of flow of a jet issuing from a submerged sluice-way and diffusing into the water downstream has been previously described $[1,2]$. The vortices grow with distance and eventually extend to the water surface through

* Principal Lectures in Fluid Mechanies, West Ham College of Teehnology, London, E. 15 the entire height of the circulation zone. The mixing produced by these vortices simultaneously causes the jet to expand until its upper limit momentarily reaches the free surface, where it divides and a stagnation point develops.

In the course of experiments to study the diffusion of the jel, it was observed that a particular kind of unsteadiness developed involving abnormal grouping of large eddies in the flow, a phenomenon which was clearly observed in semi-submerged patterns, as shown in Figure 1 a. Here, two large eddies $A$ and $B$ are indicated near the middle of the

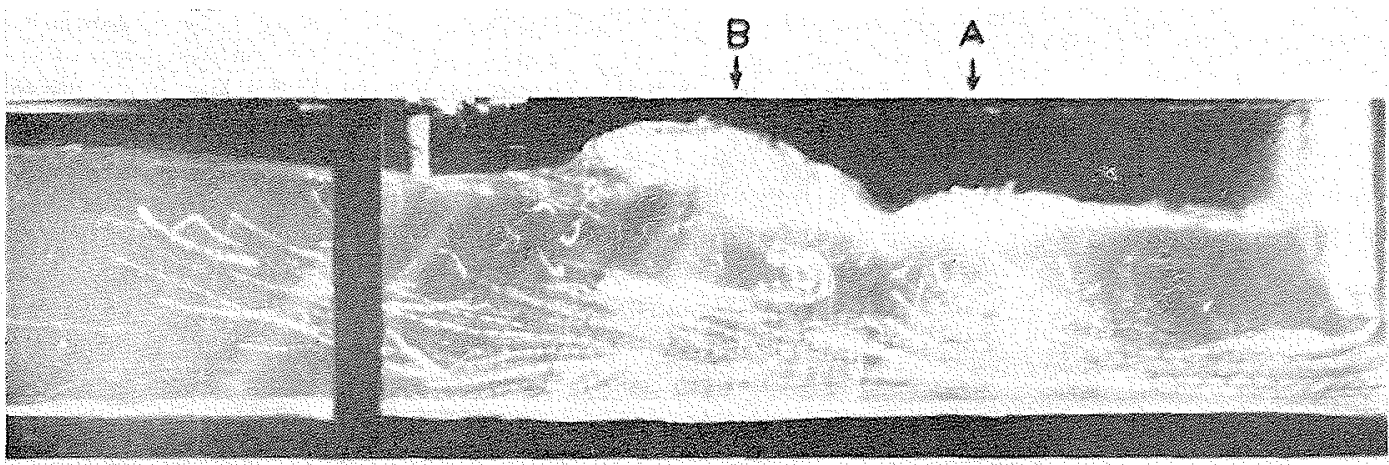

(a)



(b)

1/ Two forms of flow downstream of a submerged sluice sate. Depth upstream of sate $=7.5 \mathrm{in}$. Downstream depth $=5.4 \mathrm{in}$. Exposures: 0.2 sec.

Denx formes de l'éconlement at l'aval d'une vanne noyée. Hauteur d'eau d̀ l'amont de la banne $=7,5$ pouces. Hantteur d'eau à l'aval $=5,4$ pout ces. Temps de pose: $0,2 \mathrm{~s}$ 